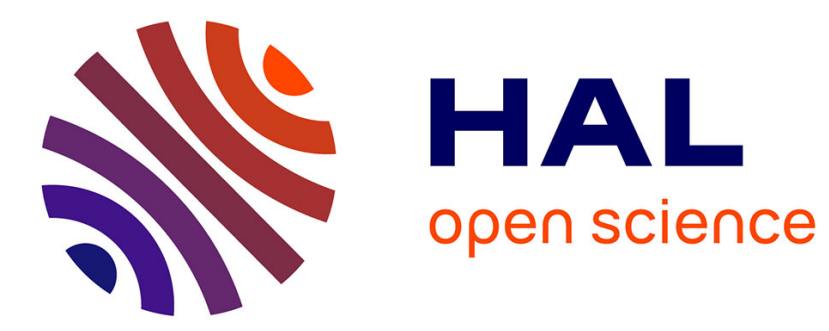

\title{
Nanoantenna for Electrical Generation of Surface Plasmon Polaritons
}

Florian Bigourdan, Jean-Paul Hugonin, François Marquier, Christophe Sauvan, Jean-Jacques Greffet

\section{To cite this version:}

Florian Bigourdan, Jean-Paul Hugonin, François Marquier, Christophe Sauvan, Jean-Jacques Greffet. Nanoantenna for Electrical Generation of Surface Plasmon Polaritons. Physical Review Letters, 2016, 116 (10), pp.106803. 10.1103/PhysRevLett.116.106803 . hal-01643230

\section{HAL Id: hal-01643230 \\ https://hal.science/hal-01643230}

Submitted on 21 Nov 2017

HAL is a multi-disciplinary open access archive for the deposit and dissemination of scientific research documents, whether they are published or not. The documents may come from teaching and research institutions in France or abroad, or from public or private research centers.
L'archive ouverte pluridisciplinaire HAL, est destinée au dépôt et à la diffusion de documents scientifiques de niveau recherche, publiés ou non, émanant des établissements d'enseignement et de recherche français ou étrangers, des laboratoires publics ou privés. 


\title{
Nanoantenna for Electrical Generation of Surface Plasmon Polaritons
}

\author{
Florian Bigourdan, Jean-Paul Hugonin, Francois Marquier, Christophe Sauvan, and Jean-Jacques Greffet ${ }^{*}$ \\ Laboratoire Charles Fabry, Institut d'Optique Graduate School, CNRS, Université Paris-Saclay, 91127 Palaiseau, France \\ (Received 20 May 2015; published 10 March 2016)
}

\begin{abstract}
Light emission by inelastic tunneling has been known for many years. Recently, this technique has been used to generate surface plasmons using a scanning tunneling microscope tip. The emission process suffers from a very low efficiency lower than a photon in $10^{4}$ electrons. We introduce a resonant plasmonic nanoantenna that allows both enhancing the power conversion to surface plasmon polaritons by more than 2 orders of magnitude and narrowing the emission spectrum. The physics of the emission process is analyzed in terms of local density of states and the efficiency of the nanoantenna to radiate surface plasmon polaritons.
\end{abstract}

DOI: 10.1103/PhysRevLett.116.106803

It has been known for decades that inelastic electron tunneling through nanoscale insulators can be used to emit light $[1,2]$ although the efficiency is very low. This field has been revived when it was observed that scanning tunneling microscopes (STM) tips can emit light with a significantly improved efficiency [3-6]. Many different theoretical studies have been devoted to this topic [7-12], and it is now well understood that the light emission process involves two steps: (i) inelastic tunneling, which generates a fluctuating electrical current, and (ii) photon emission by the time-dependent fluctuations. Yet, the efficiency remains very low-typically lower than one photon per $10^{4}$ electrons-so that inelastic tunneling has not become a practical source of light.

The fluctuating current does not only emit photons in free space, it also excites surface plasmon polaritons (SPPs) that propagate along the metal-insulator interface [13]. This possibility to generate SPPs constitutes a promising application for inelastic electron tunneling. Exciting electrically SPPs at the nanoscale is indeed a major issue in nanooptics. One of the key features of SPPs is their ability to confine light to deep subwavelength scales. However, up to now, a diffraction-limited beam has generally been used to excite them optically. Consequently, the development of plasmonic devices to manipulate light at subwavelength scales (from emission to detection) has been hampered by the impossibility of generating SPPs at the nanoscale.

Recently, inelastic tunneling by STM tips [14,15], GaAs nanowire-based light-emitting diodes [16], and impact excitation in a transistor [17] have been demonstrated as electrical sources of SPPs. Unfortunately, the efficiency of these sources remains typically lower than one plasmon per $10^{4}$ electrons. Such low values may appear to be due to some fundamental limitations. First, in the tunneling regime, light is emitted within a gap of typically $1 \mathrm{~nm}$ and quenching through nonradiative energy transfer to the nearby metal constitutes the dominant relaxation process [18-20]. Second, the emitted spectrum is imposed by the broad spectrum of the electrical intensity fluctuations. Hence, taking advantage of inelastic tunneling to realize efficient electrical sources of SPPs requires solving two problems: (i) to increase significantly the efficiency of the emission process and (ii) to control the emitted spectrum.

In this Letter, we show that the use of a resonant optical nanoantenna can circumvent these issues. We study theoretically STM light emission in the presence of a plasmonic nanoantenna under the STM tip, as depicted in Fig. 1. Previous works analyzed mainly light emission in the far field. Here, on the contrary, we focus on the fraction of light that is emitted into SPPs propagating along the gold-air interface. From the total electromagnetic field, we extract precisely the exact amount of SPP that is generated with and without antenna and we deduce the impact of the nanostructuration. The major results are a large enhancement of the power conversion to SPPs by more than 2 orders of magnitude and a narrowing of the emission spectrum, which can be controlled by the antenna geometry. Demonstrating such a huge enhancement in a system that is often thought to be plagued by quenching is promising for the development of plasmonic devices.

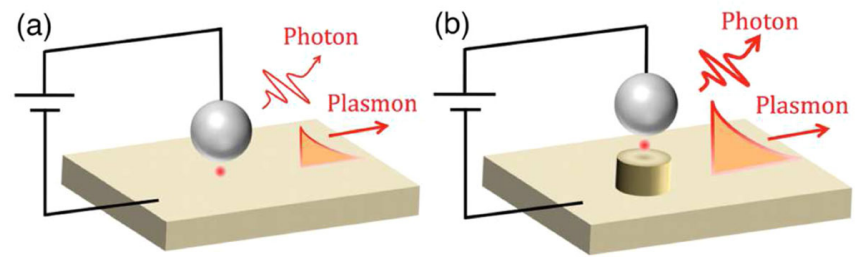

FIG. 1. Electrical generation of surface plasmon polaritons (SPPs) by inelastic electron tunneling under a STM tip. The tip apex is modeled by a tungsten sphere with a $10 \mathrm{~nm}$ radius. (a) In the reference case, the tip is positioned above a flat gold surface. (b) The reference is compared to the case where a resonant nanoantenna is placed between the tip and the surface. The antenna is a gold cylindrical nanorod (height $H=51 \mathrm{~nm}$ and diameter $D$ ). The air-gap thickness between the tip apex and the metal is kept constant, $w=2 \mathrm{~nm}$. 
Moreover, we analyze the physical mechanisms responsible for the enhanced efficiency. We report a detailed study of the nanoantenna impact on the electromagnetic local density of states (LDOS). This theoretical analysis is not limited to light emission by inelastic tunneling; it addresses fundamentals of the process of light emission assisted by an optical nanoantenna. We derive simple and general rules to design a nanoantenna able to overcome nonradiative processes. Our analysis allows one to understand recent experimental proofs of enhanced emission efficiencies [21-23].

The process of light emission by inelastic tunneling is modeled with the approach introduced in Ref. [7]. A quantum description of the electron transport provides the power spectral density of the electrical intensity fluctuations. The latter is used in a second step as a source for a radiation problem, which fully accounts for the local electromagnetic environment. The emitted optical power at a frequency $\omega$ is thus proportional to the average electrical intensity [24]. Such a linear relation between the optical power and the electrical intensity has been confirmed experimentally [27]. In the second step of the calculation, the fluctuating current can be replaced by a point dipole located at the center of the gap and polarized linearly along the vertical direction. We emphasize that this approach provides the full electromagnetic field and, hence, it accounts for both localized modes and surface waves propagating along the gold surface.

The tip apex is modeled by a tungsten sphere with a radius of $10 \mathrm{~nm}$. In order to make our analysis as independent as possible on the tip model, we compare the light emitted with and without antenna while using the same tip and gap geometry; see Fig. 1. In the tunneling regime, the tip-sample distance $w$ is of the order of 1-2 nm so that nonlocal effects may play a role. Nonlocal effects can be included in a classical model by using the Feibelman parameter, which amounts to introducing an effective distance between the surfaces [28,29]. We have checked that varying the gap width in the range $1-4 \mathrm{~nm}$ does not change the ratio of power emitted with and without antenna. Nonlocal effects are thus not expected to play a significant role. In what follows, all the calculations are performed for a $2 \mathrm{~nm}$ gap and a local model of the dielectric constants. The frequency-dependent permittivities of gold and tungsten are taken from Ref. [30]. Numerical calculations are performed with a fully vectorial method recently developed for cylindrical geometries [31].

Before studying the impact of a resonant nanoantenna on the SPP generation, let us first analyze the case of a STM tip positioned above a flat gold surface; see Fig. 1(a). We will use this flat-surface case as a reference for the study of the nanoantenna impact. We have calculated the LDOS in the center of the gap [24]. Because of the extremely small thickness of the gap, the LDOS is 5 orders of magnitude larger than the vacuum LDOS. Such a huge enhancement is mostly due to quenching, i.e., energy dissipation in the gold and the tungsten in the vicinity of the source. Only a very small fraction of light is emitted in the far field; the radiative efficiency (ratio between the power radiated in the far field and the total power) is smaller than $5 \times 10^{-4}$. Among the huge amount of dissipated power, a small fraction corresponds to the excitation of SPPs that propagate along the gold-air interface. We now focus on this SPP generation. An important result of this Letter is the rigorous and quantitative calculation of the SPP contribution in the total electromagnetic field.

In order to extract the amplitude of the SPPs that are launched around the structure, we generalize to threedimensional systems the theoretical formalism proposed in Refs. [32,33] for two-dimensional systems. The method relies on the completeness theorem of the quasinormal modes set used in the theory of lossy waveguides [34]. We use cylindrical coordinates with a $z$ axis perpendicular to the gold surface. Outside the structure, the electric field $\mathbf{E}=\left[E_{r}, E_{\theta}, E_{z}\right]$ emitted by the point source located on the axis and polarized linearly along the $z$ direction can be expanded as

$$
\mathbf{E}(r, z)=A_{s p} \mathbf{E}_{s p}^{+}(r, z)+\sum_{\sigma} A_{\sigma} \mathbf{E}_{\sigma}^{+}(r, z),
$$

where the last term corresponds to a summation over the continuum of radiation modes of the flat gold-air interface. The first term corresponds to the electric field of an outgoing SPP, which can be written as $\mathbf{E}_{s p}^{+}=$ $\left[i H_{1}^{(1)}\left(k_{s p} r\right) e_{s p, x}(z), 0, H_{0}^{(1)}\left(k_{s p} r\right) e_{s p, z}(z)\right]$. The same expansion holds for the magnetic field, with $\mathbf{H}_{s p}^{+}=$ $\left[0, i H_{1}^{(1)}\left(k_{s p} r\right) h_{s p}(z), 0\right] . H_{n}^{(1)}\left(k_{s p} r\right)$ are the Hankel functions of the first kind of order $n, k_{s p}$ is the wave vector of the flat interface SPP, and $e_{s p, x}, e_{s p, z}$, and $h_{s p}$ represent the $z$ profile of the SPP field. Their analytical expressions can be found in Ref. [24]. Note that the fields are independent of the azimuthal angle $\theta$ because of the source position and polarization. In Eq. (1), $A_{s p}$ is the modal excitation coefficient of the SPP launched along the gold-air interface. It can be calculated by using the mode orthogonality [24,34],

$$
A_{s p}=\frac{1}{N_{s p}} \iint\left(\mathbf{E}_{s p}^{-} \times \mathbf{H}-\mathbf{E} \times \mathbf{H}_{s p}^{-}\right) \cdot \mathbf{u}_{r} r d \theta d z,
$$

where the normalization constant $N_{s p}$ is given in Ref. [24]. The field of the ingoing SPP $\left(\mathbf{E}_{s p}^{-}, \mathbf{H}_{s p}^{-}\right)$can be deduced from $\left(\mathbf{E}_{s p}^{+}, \mathbf{H}_{s p}^{+}\right)$by replacing $H_{n}^{(1)}\left(k_{s p} r\right)$ by the Hankel function of the second kind. This formalism allows calculating quantitatively the fraction of light that is emitted into SPPs. It is worth noting that it can be used with any numerical method that is able to compute the total field $(\mathbf{E}, \mathbf{H})$ emitted by a source. 


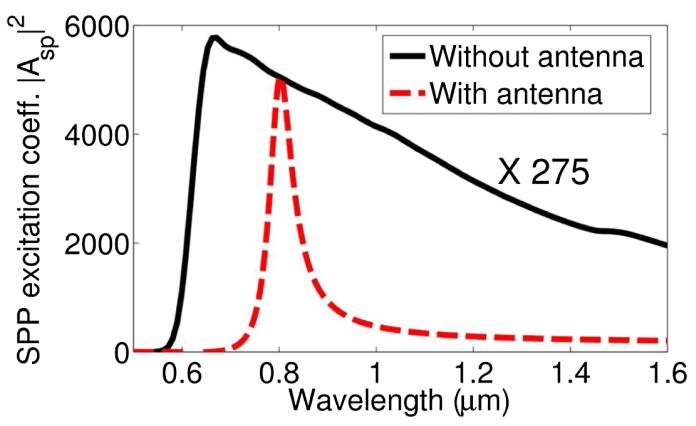

FIG. 2. SPP excitation coefficient without antenna (solid black line) and with antenna (dashed red line). $\left|A_{s p}\right|^{2}$ is calculated with Eq. (2). The presence of the antenna strongly modifies the emission spectrum and enhances the SPP generation by a factor of 275 at the resonance frequency. The diameter of the gold nanorod is $D=25 \mathrm{~nm}$.

We first calculate the SPP amplitude in the flat-surface case of Fig. 1(a). The spectrum of the SPP excitation coefficient $\left|A_{s p}\right|^{2}$ is shown with the solid curve in Fig. 2. Note that SPPs are generated over a broad spectrum, which is directly related to the broad spectrum of the fluctuating current. We have used for the calculation a voltage value that is typically used in experiments $(V=2.07 \mathrm{~V})$ and that corresponds to a cutoff wavelength of photon emission $\lambda_{c}=600 \mathrm{~nm}$ [24].

We now turn to the analysis of SPP generation in the presence of a resonant plasmonic antenna. We place a gold nanorod (varying diameter $D$ and height $H=51 \mathrm{~nm}$ ) under the STM tip. The spectrum of the SPP excitation coefficient is displayed in Fig. 2. The emission is deeply modified by the resonance of the plasmonic antenna. The first salient feature is the enhancement by a factor 275 of the SPP excitation coefficient. A second important feature is that the spectrum now presents a narrow peak. This result suggests that the emission spectrum can be tuned by changing the resonance spectral characteristics. We report in Fig. 3 the enhancement factor of SPP excitation for three

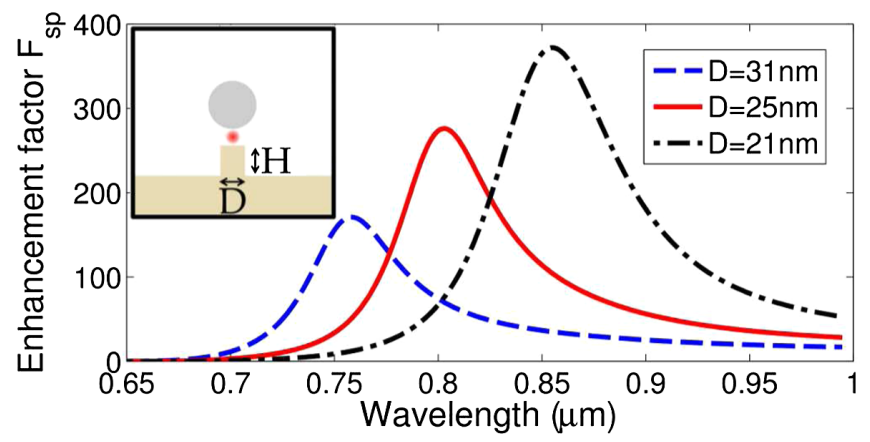

FIG. 3. Enhancement factor of SPP excitation, defined as $\left|A_{s p}^{\text {ant }}\right|^{2} /\left|A_{s p}^{0}\right|^{2}$. The emission frequency can be controlled by varying the nanorod diameter: $D=31 \mathrm{~nm}$ (dashed blue curve), $D=25 \mathrm{~nm}$ (solid red curve), and $D=21 \mathrm{~nm}$ (dash-dotted black curve). The cylinder height is fixed to $H=51 \mathrm{~nm}$. different values of the nanorod diameter. The enhancement factor is defined as the ratio of the SPP excitation coefficient with and without antenna, $F_{s p}=\left|A_{s p}^{\text {ant }}\right|^{2} /$ $\left|A_{s p}^{0}\right|^{2}$. The spectrum blueshifts as the diameter increases, in agreement with the behavior of the nanorod resonance frequency as a function of the diameter-over-height ratio [35]. The emission frequency can be controlled while keeping an enhancement factor larger than 100. Using a gold tip provides the same results [24].

Before analyzing in detail the enhancement mechanism, we discuss qualitatively the role of the antenna. Figure 4(a) displays a map of the field emitted by the source [36]. We observe an intense hot spot localized in the vicinity of the source. This intense field localized in the gap is similar to the one produced by the dipole in the flat-surface case, as shown in Fig. 4(c) [24]. Interestingly, Fig. 4(a) also shows an intense field around the edges of the cylinder. This part of the total field can be associated to the nanorod mode displayed in Fig. 4(b). The total field radiated by the source in the presence of the antenna can thus be seen as a superposition of the nanorod mode and the gap field observed in the flat-surface case. The resonant mode is expected to modify the LDOS and to offer a new decay channel. Is the new channel able to compete with the huge amount of quenching that dominates the relaxation process? We now report a detailed analysis that answers this question.

First, we plot in Fig. 5(a) the LDOS spectrum with antenna normalized by that without antenna for different nanorod diameters. The LDOS is enhanced only within the
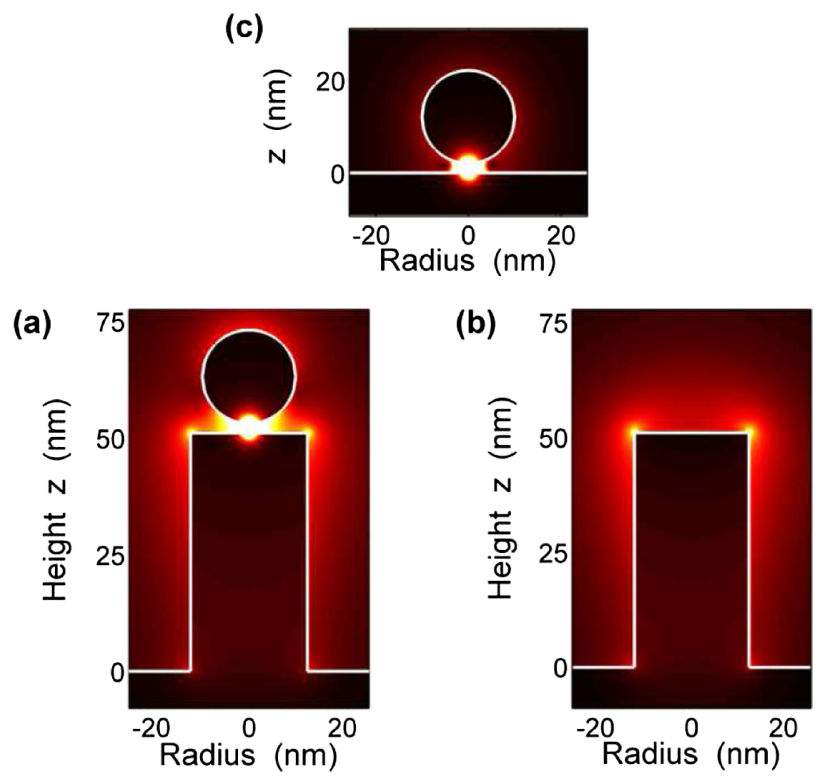

FIG. 4. (a) Distribution of the emitted field $|E|$ in the presence of a gold nanorod below the tip at $\lambda=806 \mathrm{~nm}$ [36]. (b) Distribution of $|E|$ for the mode of the antenna, i.e., a nanorod over a gold surface without tungsten sphere. (c) Distribution of the emitted field $|E|$ without gold nanorod at $\lambda=806 \mathrm{~nm} \mathrm{[36].}$ 
bandwidth of the antenna resonance, leading to the narrow spectral feature of the emission. The presence of the antenna increases the LDOS by a factor of 1.3-1.65 at resonance. This result evidences that the contribution of the antenna mode to the LDOS is of the same order of magnitude as the quenching contribution. At such small distances from the metal surface, the LDOS due to quenching is huge, of the order of $10^{5}$ times the vacuum LDOS. Our calculations show that the LDOS of the mode is also 5 orders of magnitude larger than the vacuum LDOS. The new decay channel offered by the antenna is thus able to compete with the quenching. The LDOS increase is a necessary but not sufficient condition to explain the 2 orders of magnitude enhancement of the SPP excitation coefficient. A second important parameter is the antenna ability to radiate.

We introduce the plasmonic efficiency defined by the SPP excitation coefficient normalized by the total power delivered by the source. This ratio is denoted $\eta_{s p}^{0}=$ $\left|A_{s p}^{0}\right|^{2} / P_{\text {tot }}^{0}$ for a flat surface and $\eta_{s p}^{\text {ant }}=\left|A_{s p}^{\text {ant }}\right|^{2} / P_{\text {tot }}^{\text {ant }}$ in the presence of the antenna. The enhancement factor shown in Fig. 3 can be written as

$$
F_{s p}=\frac{\left|A_{s p}^{\mathrm{ant}}\right|^{2}}{\left|A_{s p}^{0}\right|^{2}}=\frac{P_{\mathrm{tot}}^{\mathrm{ant}}}{P_{\mathrm{tot}}^{0}} \frac{\eta_{s p}^{\mathrm{ant}}}{\eta_{s p}^{0}}
$$

The first term is the LDOS enhancement at the source position and the second term is the plasmonic efficiency enhancement. From Fig. 5(a), we have seen that the antenna increases the LDOS by a factor of 1.5. It follows that the enhancement of the SPP excitation shown in Fig. 3 can be mostly attributed to an increase of the plasmonic efficiency $\eta_{s p}$. Figure 5(b) shows the plasmonic efficiency with and
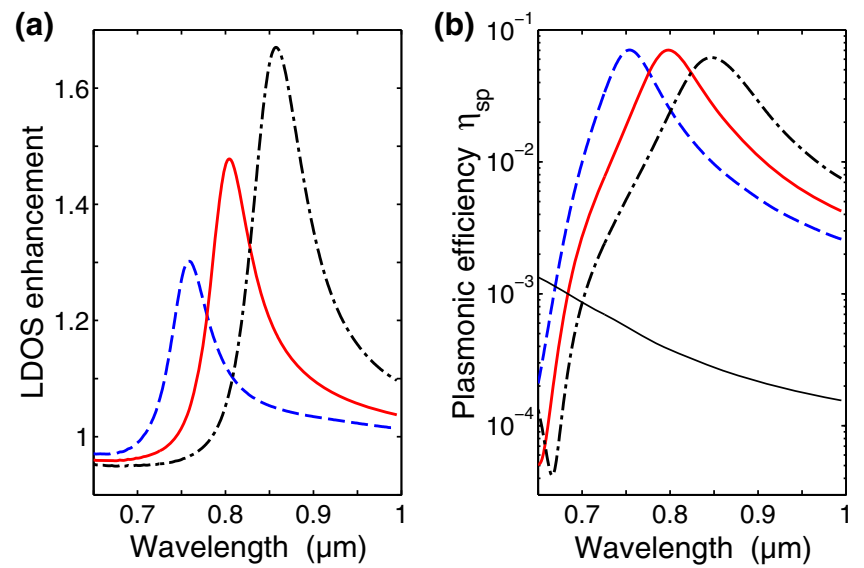

FIG. 5. Analysis of the enhancement mechanism. (a) Ratio of the LDOS with and without antenna for three nanorod diameters: $D=31 \mathrm{~nm}$ (dashed blue curve), $D=25 \mathrm{~nm}$ (solid red curve), and $D=21 \mathrm{~nm}$ (dash-dotted black curve). (b) Plasmonic efficiency $\eta_{s p}$ for the same nanorods and for the flat-surface case (thin black line). The antenna increases the plasmonic efficiency by more than 2 orders of magnitude. without antenna. In the flat-surface case, it is of the order of $5 \times 10^{-4}$ (same order as the radiative efficiency, i.e., the ratio between the power radiated in the far field and the total power). In the presence of the antenna, the plasmonic efficiency increases by 2 orders of magnitude up to 0.07 . This dramatic improvement is essentially due to the much larger dipole moment of the plasmonic resonator. Note that the radiative efficiency enhancement is of the same order of magnitude.

This theoretical analysis allows us to derive simple and general rules to design a nanoantenna able to overcome the quenching. The antenna should support a mode that presents both (i) a hot spot, which generates a LDOS as large as the quenching contribution, and (ii) a large dipole moment in order to radiate efficiently.

In summary, we have analyzed the SPP emission process by inelastic electron tunneling between a STM tip and a metallic surface. We have rigorously calculated the SPP contribution among the total amount of emitted light. The very low efficiency usually observed can be attributed to nonradiative decay channels in the metal (quenching) which are very efficient in the distance regime relevant for tunneling. By inserting a resonant plasmonic nanoantenna, it is possible to introduce a contribution to the electromagnetic LDOS that has the same order of magnitude $\left(\sim 10^{5}\right.$ larger than the vacuum LDOS). As the nanoantenna has a much better ability to radiate SPPs than the bare system, the power emitted into SPPs can be enhanced by more than 2 orders of magnitude. This huge enhancement of SPP excitation comes with a narrowing of the emission spectrum and hence a possibility to control the emission frequency with the antenna geometry. The directivity of SPP emission could be engineered by using a small array of antennas. These properties pave the way towards the design of integrated electrical sources of SPPs with controlled emission frequencies.

J. J. G. is a senior member of IUF. J. J. G. acknowledges the support of the SAFRAN-IOGS chair on Ultimate Photonics.

*Corresponding author. jean-jacques.greffet@institutoptique.fr

[1] J. Lambe and S. L. McCarthy, Phys. Rev. Lett. 37, 923 (1976).

[2] J. Kirtley, T. N. Theis, and J. C. Tsang, Phys. Rev. B 24, 5650 (1981).

[3] J. K. Gimzewski, J. K. Sass, R. R. Schlitter, and J. Schott, Europhys. Lett. 8, 435 (1989).

[4] R. Berndt, J. K. Gimzewski, and P. Johansson, Phys. Rev. Lett. 67, 3796 (1991).

[5] D. Walmsley, T.-S. Tan, and P. Dawson, Surf. Sci. 572, 497 (2004).

[6] K. Perronet, L. Barbier, and F. Charra, Phys. Rev. B 70, 201405 (2004). 
[7] D. Hone, B. Mühlschlegel, and D. J. Scalapino, Appl. Phys. Lett. 33, 203 (1978).

[8] B. Laks and D. L. Mills, Phys. Rev. B 20, 4962 (1979).

[9] P. Johansson, R. Monreal, and P. Apell, Phys. Rev. B 42, 9210 (1990).

[10] B. N. J. Persson and A. Baratoff, Phys. Rev. Lett. 68, 3224 (1992).

[11] J. Aizpurua, S. P. Apell, and R. Berndt, Phys. Rev. B 62, 2065 (2000).

[12] J. T. Lü, R. B. Christensen, and M. Brandbyge, Phys. Rev. B 88, 045413 (2013).

[13] A. D. Boardman, Electromagnetic Surface Modes (John Wiley, New York, 1982).

[14] P. Bharadwaj, A. Bouhelier, and L. Novotny, Phys. Rev. Lett. 106, 226802 (2011).

[15] T. Wang, E. Boer-Duchemin, Y. Zhang, G. Comtet, and G. Dujardin, Nanotechnology 22, 175201 (2011).

[16] P. Fan, C. Colombo, K. C. Huang, P. Krogstrup, J. Nygård, A. Fontcuberta i Morral, and M. L. Brongersma, Nano Lett. 12, 4943 (2012).

[17] P. Rai, N. Hartmann, J. Berthelot, J. Arocas, G. Colas des Francs, A. Hartschuh, and A. Bouhelier, Phys. Rev. Lett. 111, 026804 (2013).

[18] J. M. Wylie and J. E. Sipe, Phys. Rev. A 30, 1185 (1984).

[19] P. Anger, P. Bharadwaj, and L. Novotny, Phys. Rev. Lett. 96, 113002 (2006).

[20] S. Kühn, U. Håkanson, L. Rogobete, and V. Sandoghdar, Phys. Rev. Lett. 97, 017402 (2006).

[21] K. J. Russell, T. L. Liu, S. Cui, and E. L. Hu, Nat. Photonics 6, 459 (2012).

[22] G. M. Akselrod, C. Argyropoulos, T. B. Hoang, C. Ciraci, C. Fang, J. Huang, D. R. Smith, and M. H. Mikkelsen, Nat. Photonics 8, 835 (2014).
[23] J. Kern, R. Kullock, J. Prangsma, M. Emmerling, M. Kamp, and B. Hecht, Nat. Photonics 9, 582 (2015).

[24] See Supplemental Material at http://link.aps.org/ supplemental/10.1103/PhysRevLett.116.106803 for more details on the modeling of inelastic tunneling, the LDOS of the flat-surface case, and the theoretical formalism used to calculate the SPP generation, and for Refs. [25-26].

[25] S. Divitt, P. Bharadwaj, and L. Novotny, Opt. Express 21, 27452 (2013).

[26] O. Lozan, M. Perrin, B. Ea-Kim, J. M. Rampnoux, S. Dilhaire, and P. Lalanne, Phys. Rev. Lett. 112, 193903 (2014).

[27] N. L. Schneider, G. Schull, and R. Berndt, Phys. Rev. Lett. 105, 026601 (2010).

[28] P. J. Feibelman, Prog. Surf. Sci. 12, 287 (1982).

[29] T. V. Teperik, P. Nordlander, J. Aizpurua, and A. G. Borisov, Phys. Rev. Lett. 110, 263901 (2013).

[30] E. D. Palik, Handbook of Optical Constants of Solids (Academic, New York, 1985).

[31] F. Bigourdan, J. P. Hugonin, and P. Lalanne, J. Opt. Soc. Am. A 31, 1303 (2014).

[32] P. Lalanne, J. P. Hugonin, and J. C. Rodier, Phys. Rev. Lett. 95, 263902 (2005).

[33] P. Lalanne, J. P. Hugonin, H. T. Liu, and B. Wang, Surf. Sci. Rep. 64, 453 (2009).

[34] A. W. Snyder and J. D. Love, Optical Waveguide Theory (Chapman and Hall, New York, 1983).

[35] C. F. Bohren and D. R. Huffman, Absorption and Scattering of Light by Small Particles (Wiley, New York, 1983).

[36] To avoid the field divergence due to the point source, we have subtracted, in air, the field emitted by the source alone without tungsten sphere and without antenna. 